\title{
Proposição de uma metodologia preditiva para avaliação do efeito caking em diferentes tipos de açúcares comerciais
}

\section{A proposed predictive methodology for the evaluation of caking effect on different types of commercial sugars}

\author{
Rodrigo Condotta ${ }^{1 *}$ (1D, Pamela Marques da Silva ${ }^{1}$ \\ ${ }^{1}$ Centro Universitário FEI, Departamento de Engenharia Química, São Bernardo do Campo/SP - Brasil
}

*Corresponding Author: Rodrigo Condotta, Centro Universitário FEI, Departamento de Engenharia Química, Av. Humberto de Alencar Castelo Branco, 3972, CEP: 09850-901, São Bernardo do Campo/SP - Brasil, e-mail: rcondotta@fei.edu.br

Cite as: Condotta, R., \& Silva, P. M. (2020). A proposed predictive methodology for the evaluation of caking effect on different types of commercial sugars. Brazilian Journal of Food Technology, 23, e2019076.

https://doi.org/10.1590/1981-6723.07619

\begin{abstract}
Resumo
Este trabalho apresenta os resultados preliminares de uma metodologia experimental alternativa em desenvolvimento para se prever o efeito caking comumente observados em materiais particulados durante o seu período de armazenamento. Tal metodologia se fundamenta em: 1) princípio da superação da tensão de cisalhamento máxima para promover o movimento das partículas num leito particulado consolidado; 2) que essa tensão máxima é função das propriedades físicas e da natureza química das partículas que constituem o leito; 3) que a técnica de prorating é uma técnica comprovada e frequentemente utilizada nos ensaios de cisalhamento para correções de possíveis desvios; 4) que a tensão de cisalhamento estática é dependente do tempo de contato das partículas, cujo comportamento pode ser descrito pelo modelo de fricção sólida de Dieterich-Rice-Ruina. Essa metodologia preditiva visa ao estudo da evolução do coeficiente de fricção estático com o tempo, por meio de ensaios de cisalhamento do tipo stop-and-go, segundo o modelo de Dieterich-Rice-Ruina. Posteriormente, o incremento na tensão de cisalhamento em função do tempo é aplicado na técnica de prorating, para, a partir de um plano de ruptura instantâneo, estimar o plano de ruptura temporal. Foram realizados testes com quatro tipos de açúcares comerciais, para os quais os valores dos planos de ruptura temporais, obtidos experimentalmente, e dos planos teóricos, estimados segundo o método preditivo proposto, se mostraram inicialmente satisfatórios para os curtos períodos de tempo ensaiados neste estudo preliminar, podendo ser ampliado para períodos maiores.
\end{abstract}

Palavras-chave: Açúcar; Cisalhamento granular; Plano de ruptura temporal; Prorating, caking.

\begin{abstract}
This paper presents preliminary results of an alternative experimental methodology under development to predict the caking effect often observed in bulk solids after time storage. This methodology is based on 1) the principle that the particle is sheared after the shear stress overcome a maximum value; 2 ) the maximum shear stress depends on the particles physical properties and their chemical nature; 3 ) Prorating is a well-known and often used empirical
\end{abstract}


procedure to minimize the scatter at shear tests; and 4) that the static friction coefficient depends on particle-particle contact time and could be described by the phenomenological state-and-rate model formulated by Dieterich, Rice, and Ruina. The proposed methodology aims to evaluate the increasing of the static coefficient of friction with rest time, through "stop-and-go" shear experiments and according to Dieterich, Rice and Ruina model. Finally, the increase in shear stress with time is used to extrapolate the instantaneous yield locus into a predicted time yield locus, using the prorating technique We tested four types of commercial sugar and the results of time yield locus, obtained from experiments, have seemed similar to those predicted using the proposed methodology. We obtained satisfactory results for the short rest times tested in this preliminary study, which could be extended to long periods.

Keywords: Sugar; Granular shear; Time yield locus; Prorating, caking.

\section{Introdução}

O açúcar é um produto de consumo básico comumente utilizado por indústrias do setor alimentício como matéria-prima básica de bebidas, produtos de panificação, confeitos etc., além de ser uma commodity essencial. O açúcar proveniente da cana-de-açúcar é responsável por $70 \%$ da produção mundial, sendo o Brasil o principal produtor (Brasil, 2017).

Por ser um produto bastante utilizado no dia a dia, o comportamento do açúcar é bem conhecido. A formação de aglomerados ou mesmo o empedramento total de uma embalagem de açúcar são fenômenos frequentemente observados no ambiente doméstico. Entretanto, essa quantidade é ínfima quando comparada com a capacidade industrial, em que esses mesmos problemas nessa escala são preocupantes (Silva \& Condotta, 2017).

Muitos outros materiais nas indústrias alimentícias, farmacêuticas e química, que são empregados em forma de pó, também podem apresentar um comportamento semelhante. Durante o período de armazenamento, um pó de boa fluidez pode ter a capacidade de escoar reduzida devido a modificações físico-químicas que podem ocorrer. $\mathrm{O}$ aumento das interações entre as partículas que constituem o pó, denominada de coesão, é a principal causa (Condotta \& Machado, 2015). O aumento dessa coesão durante o tempo de armazenamento do material é, muitas vezes, referida como um dos grandes problemas ao se manusear produtos pulverulentos, resultando na formação de caking (Rogé \& Mathlouthi, 2003).

Geralmente, esse fenômeno é atribuído à umidade ambiente, que acaba sendo adsorvida nas superfícies das partículas, à umidade do material nos interstícios (Chávez Montes et al., 2011), à temperatura (Hartmann \& Palzer, 2011) e às intensificações das interações das partículas do material ao longo do tempo, como os fenômenos de cristalização e sinterização (Mehos, 2016).

Entretanto, a compreensão exata dos mecanismos que levam à formação de caking que afeta uma grande variedade de materiais particulados ainda é um grande desafio para a indústria. Apesar de não existir uma solução comum a todos os segmentos, a predição da aptidão ao efeito caking dos materiais é vital para o bom funcionamento do processo (Calvert et al., 2013).

Fürll \& Hoffmann (2013) citam que tem sido possível determinar a dependência das propriedades de escoamento com o tempo de consolidação de materiais sólidos por meio dos ensaios de obtenção do plano temporal de ruptura desenvolvido por Jenike (1964), permitindo o dimensionamento de silos que podem ser descarregados após certo tempo de armazenamento desse tipo de materiais. Entretanto, poucos trabalhos relacionando a granulometria do material com essa dependência do tempo têm sido realizados. Além disso, nenhum deles elucida como essas três características essenciais se relacionam entre si: granulometria, tempo de consolidação e interações interparticulares que atuam diretamente no fenômeno caking.

Diante da problemática exposta, Zafar et al. (2017) apresentam uma revisão sobre os equipamentos mais empregados para avaliar o efeito caking e concluem que estes fornecem um melhor entendimento empírico 
do fenômeno, mas são limitados na capacidade de predizê-lo, e alegam que os equipamentos capazes de fazer tal predição ainda se encontram no "estado da arte".

Assim, baseando-se no modelo de fricção sólida de Dieterich-Rice-Ruina (Dieterich, 1979), originalmente proposto para estudar a dependência do fenômeno de friç̧ão com o tempo, e com o auxílio de um equipamento capaz de quantificar as forças de atrito e de coesão de materiais particulados (célula de cisalhamento), este trabalho apresenta os resultados preliminares de uma metodologia em desenvolvimento para prever o fenômeno caking de materiais pulverulentos quando submetidos a períodos de armazenamento.

\section{Síntese bibliográfica}

\subsection{Fluidez dos materiais particulados}

Alguns fatores associados ao produto em pó que podem influenciar a fluidez incluem: distribuição do tamanho de partículas e umidade do produto (Fürll \& Hoffmann, 2013); rugosidade de superfície e formato de partícula (Campos \& Ferreira, 2013); ou, ainda, fatores relacionados ao ambiente de estocagem (Fitzpatrick \& Ahrné, 2005).

$\mathrm{O}$ fenômeno conhecido como caking é um processo indesejado que compromete a fluidez dos materiais particulados. A intensidade desse fenômeno depende de como tais fatores físicos mencionados se associam, resultando em novas interações partícula-partícula, formando aglomerados e aumentado a coesão do sistema (Calvert et al., 2013).

Essas interações se tornam mais intensas quando o material é comprimido, implicando um aumento do número de contato, ou por deformação da partícula em razão dessa compressão, resultando numa maior área superficial de contato entre as partículas (Johanson, 2009).

Ao se tratar de materiais úmidos, essas interações interparticulares podem ser ainda mais intensas. Pontes capilares devidas à umidade presente nos interstícios e o desenvolvimento de novas pontes capilares em outros pontos de contato resultante da migração dessa umidade (atividade da água) acentuam a coesão do meio. Neste aspecto, os pós alimentícios são os mais imprevisíveis em termos de escoabilidade, em razão do grande número de fatores que podem mudar significativamente a "reologia" do escoamento (Juliano \& Barbosa-Cánovas, 2010).

Assim sendo, independentemente do mecanismo que tenha ocasionado o incremento das forças coesivas de um material pulverulento, a estimativa da energia necessária para desfazer essas interações e devolver ao material sua fluidez original é o que propõe a avaliar um estudo de caking. Dentre as poucas técnicas existentes capazes de mensurar a coesão desenvolvida em um sistema particulado, os testes de cisalhamento são os mais indicados (Zafar et al., 2017).

\subsection{Teste de cisalhamento}

O teste pioneiro para determinar as propriedades de fluxo de materiais particulados é a célula de Jenike (1964). Esse teste permite determinar o plano de ruptura (yield locus) de um leito constituído por partículas, sob diferentes condições de consolidação, e também a chamada função de fluxo do material. Ressalta-se que todos os pontos do plano de ruptura são obtidos em ensaios distintos, podendo levar a erros experimentais. Desta forma, visando à mais precisão dos experimentos, foram desenvolvidos diferentes equipamentos ao longo das últimas décadas que permitem determinar um plano de ruptura completo em apenas um ensaio: célula anular de Schulze, célula de Pearsch e o reômetro de pó FT-4 (Freeman). Um resultado típico de um ensaio de cisalhamento granular completo, utilizando qualquer um desses equipamentos, é ilustrado pela Figura 1. 


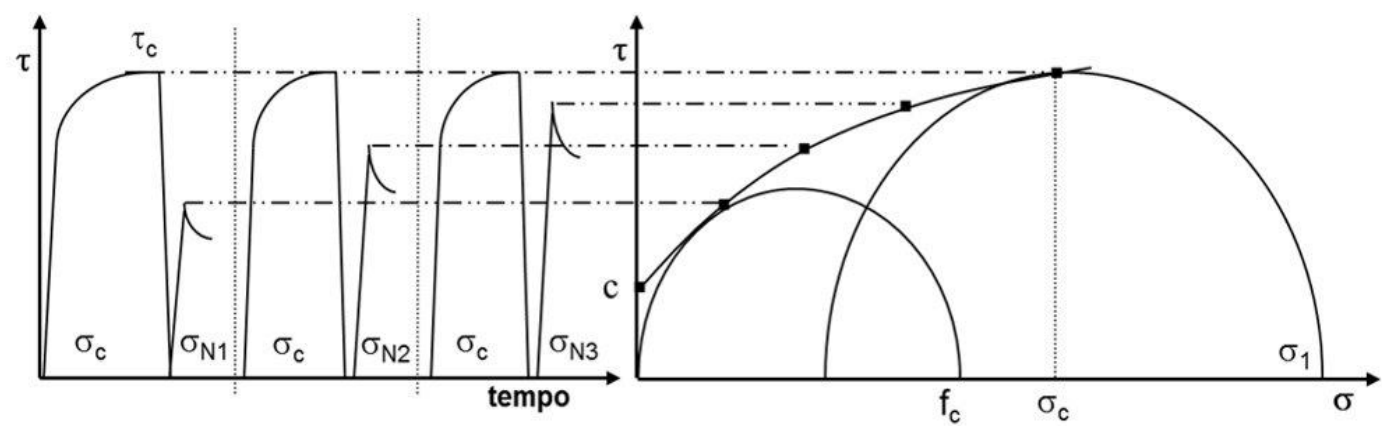

Figura 1. Resultado típico de um ensaio de cisalhamento para a determinação do plano de ruptura instantâneo, segundo a norma ASTM D6128-14 (Condotta, 2017).

Segundo a metodologia de Jenike (1964), após a determinação do plano de ruptura de um meio granular, ou seja, de um conjunto de forças de cisalhamento necessárias para promover o escoamento inicial (ruptura) do meio granular, submetido precedentemente a um estado de compressão/consolidação $\left(\sigma_{C}\right)$, os seguintes parâmetros físicos podem ser obtidos: (i) ângulo de atrito interno $\left(\varphi_{i}\right)$; (ii) ângulo efetivo de atrito interno: $\left(\varphi_{e f}\right)$; (iii) coesão $(c)$; (iv) tensão máxima de consolidação no plano principal $\left(\sigma_{l}\right)$; e (v) tensão não confinada de deslizamento $(f c)$ (Schwedes, 2003).

$\mathrm{O}$ ângulo efetivo de atrito interno $\left(\varphi_{\mathrm{ef}}\right)$ é um ângulo atribuído por Jenike como sendo uma grandeza proveniente de uma combinação entre os fenômenos puro de fricção (atrito), dado pelo ângulo de atrito interno $\left(\varphi_{i}\right)$, e de coesão $(c)$ do meio granular (Condotta, 2017).

Finalmente, a realização de diversos ensaios de cisalhamento levará à confecção de alguns planos de ruptura que, por sua vez, resultará na função de fluxo $(F F)$ do sistema particulado, definindo sua aptidão ao escoamento (Schwedes, 2003; Lopes-Neto et al., 2007).

Desta forma, é possível analisar as condições em que um determinado material, que foi previamente armazenado/estocado (consolidado), terá o escoamento restabelecido.

Entretanto, todas os efeitos mencionados anteriormente são obtidos no chamado teste de cisalhamento instantâneo (ou imediato), no qual o material é colocado em fluxo (ruptura) logo após a etapa de consolidação. Mas esses mesmos efeitos também podem ser avaliados levando-se em consideração a variável tempo, quando o material permanece armazenado em condições estabelecidas por diferentes períodos. Essa análise é realizada por meio do chamado teste de cisalhamento temporal.

\subsection{Teste de cisalhamento temporal}

O teste de cisalhamento temporal visa avaliar a evolução da coesão e da função de fluxo dos materiais particulados em função do tempo em que este permaneceu em repouso (armazenagem, estocagem), sob certas condições (consolidação, umidade, temperatura).

A principal dificuldade desse teste está em repetir com exatidão as mesmas condições do material durante a determinação de cada ponto de ruptura, uma vez que cada ponto do plano de ruptura temporal (time yield locus) é obtido individualmente. Se o tempo de exposição for muito longo, o material na célula poderá sofrer oscilações de temperatura e umidade, diferentes das condições reais às quais é submetido dentro da embalagem.

Partindo-se do princípio de que a natureza do material não se modifica e, para materiais insolúveis em água, em que a superfície não sofre alterações (dissolução), adota-se a hipótese de que o fenômeno puro de fricção não se altera, apenas a coesão a ser rompida para iniciar o cisalhamento sofre um acréscimo de intensidade com o tempo. Neste caso, a técnica simplificada consiste em determinar apenas um ponto de 
ruptura temporal e, utilizando-se a mesma inclinação do plano de ruptura instantâneo, obtém-se um novo plano de ruptura temporal completo que deve passar por esse único ponto experimental obtido.

Segundo as normas D6773-16 e D6128-14 (American Society for Testing and Materials, 2014, 2016), existem duas maneiras de proceder à determinação do ponto de ruptura temporal. Uma delas consiste em, do ponto de vista conceitual, deixar a amostra em repouso na célula de cisalhamento, uma vez atingido o regime permanente do estado de consolidação. Desta forma, a amostra ficará exatamente sob as tensões normal e tangencial em que foi condicionada. Entretanto, o equipamento permanecerá inutilizado durante o período em que a amostra permanecer em repouso.

A segunda maneira de proceder a esse tipo de teste é retirar o conjunto contendo a amostra (amostra + base do equipamento) e, em um dispositivo apropriado, recriar as mesmas condições de tensões desenvolvidas pelo material durante sua consolidação, deixando o equipamento disponível para uso. Entretanto, não é possível restabelecer a tensão de cisalhamento previamente imposta à amostra e, para recriar as mesmas condições do estado de consolidação, a tensão normal principal $\left(\sigma_{1}\right)$ será, então, aplicada à amostra que permanecerá em repouso.

\subsection{Técnica de prorating}

Conforme mencionado, a determinação do plano de ruptura instantâneo (ou imediato) envolve sucessivas etapas de consolidações sob uma mesma tensão $\left(\sigma_{c}\right)$, seguidas de etapas de cisalhamento (deslizamento) para determinar cada ponto de ruptura.

Entretanto, algumas vezes o material não retorna à mesma condição de consolidação anterior, podendo resultar em pequenos desvios no ponto de ruptura, determinado a partir desse novo estado de consolidação.

Para corrigir essa flutuação de valores, utiliza-se a técnica de prorating, bem descrita e validada na literatura (American Society for Testing and Materials, 2014, 2016), segundo a Equação 1:

$\tau_{\exp i}^{\prime}=\tau_{\exp i}\left(\frac{\tau_{C \text { médio }}}{\tau_{C \exp i}}\right)$

Em resumo, a técnica de prorating consiste em corrigir o valor da tensão de cisalhamento do ponto de ruptura ensaiado $\left(\tau_{\text {exp } i}\right)$, segundo o estado real em que a amostra foi consolidada $\left(\tau_{c \text { exp }}\right)$, para um estado de consolidação médio $\left(\tau_{c}\right.$ médio $)$ de todas as etapas de consolidação ao longo do experimento.

\subsection{Modelo de Dieterich-Rice-Ruina}

O fenômeno de fricção sólido-sólido para materiais rígidos de diversas naturezas, com diferentes velocidades de fricção $\left(V_{0}\right.$ e $\left.V\right)$ e tempos de contato $(\phi)$, foi estudado por Rice \& Ruina (1983) e Dieterich \& Conrad (1984). Suas principais evidências foram: (i) que o coeficiente de friç̧ão dinâmico $\left(\mu_{S S}=\tau_{c} / \sigma_{c}\right)$ diminui com a velocidade de deslizamento; (ii) o coeficiente de friç̧ão estático aumenta com o tempo de contato entre os dois corpos sólidos; (iii) uma perturbação na velocidade de deslizamento será absorvida após certa distância $(D)$, equacionada na Expressão 2:

$\mu_{S S}=\mu_{0}+a \cdot \ln \left(\frac{V}{V_{0}}\right)+b \cdot \ln \left(\frac{V_{0} \phi}{D}\right)$

onde o tempo de contato em regime transiente é expresso pela Equação 3:

$\frac{d \phi}{d t}=1-\frac{V \phi}{D}$

Para um cisalhamento em regime permanente, o tempo de contato é dado pela Equação 4:

$\phi=\phi_{S S}=\frac{D}{V}$ 
A expressão original 2 pode, então, ser simplificada para o regime estacionário, conforme a Expressão 5:

$\mu_{S S}=\mu_{0}+(a-b) \cdot \ln \left(\frac{V}{V_{0}}\right)$

Lubert et al. (2001) e De Ryck et al. (2003) realizaram experimentos para determinar os parâmetros $a$ e $b$ utilizando esferas de vidro e sílica gel, respectivamente. Tais experimentos consistiram em interromper e reiniciar o cisalhamento (ensaios denominados stop-and-go), obtendo picos do coeficiente de atrito estático $\left(\mu_{\text {máx }}\right) \operatorname{logo}$ ao reiniciar o experimento. Os autores verificaram que esse aumento no coeficiente de atrito era função do tempo em que o material permaneceu em repouso $(\phi)$, mesmo reiniciando o cisalhamento na mesma velocidade de deslocamento. Essa variação é mostrada pela Equação 6:

$\mu_{\max }-\mu_{S S} \propto b \cdot \ln (\phi)$

Dividindo-se as tensões de cisalhamento do regime estacionário $(\tau$ regime $)$ e da retomada do cisalhamento $(\tau$ retomada $)$ pela tensão normal aplicada nos ensaios $\left(\sigma_{n}\right)$, têm-se os coeficientes de atrito dinâmico $\left(\mu_{s s}\right) \mathrm{e}$ estático $\left(\mu_{\text {máx }}\right)$, respectivamente. A Figura 2 representa o incremento da tensão de cisalhamento e, consequentemente, do coeficiente de atrito estático em função de que a amostra permaneceu em repouso.

Sendo o comportamento da variação do coeficiente de atrito descrita pelo modelo de Dieterich-Rice-Ruina, infere-se que após a determinação do parâmetro $b$ da Equação 6, por meio de ensaios de curtos períodos de tempo, uma extrapolação para períodos de tempos mais longos possa ser, então, realizada.

Uma vez extrapolados os coeficientes de atrito estático, as tensões máximas de cisalhamento podem ser estimadas, e sabendo que esse aumento na tensão de cisalhamento é resultado das forças coesivas que se intensificaram ao longo do período de repouso da amostra, tem-se, então, uma estimativa do efeito caking para esses longos períodos extrapolados.

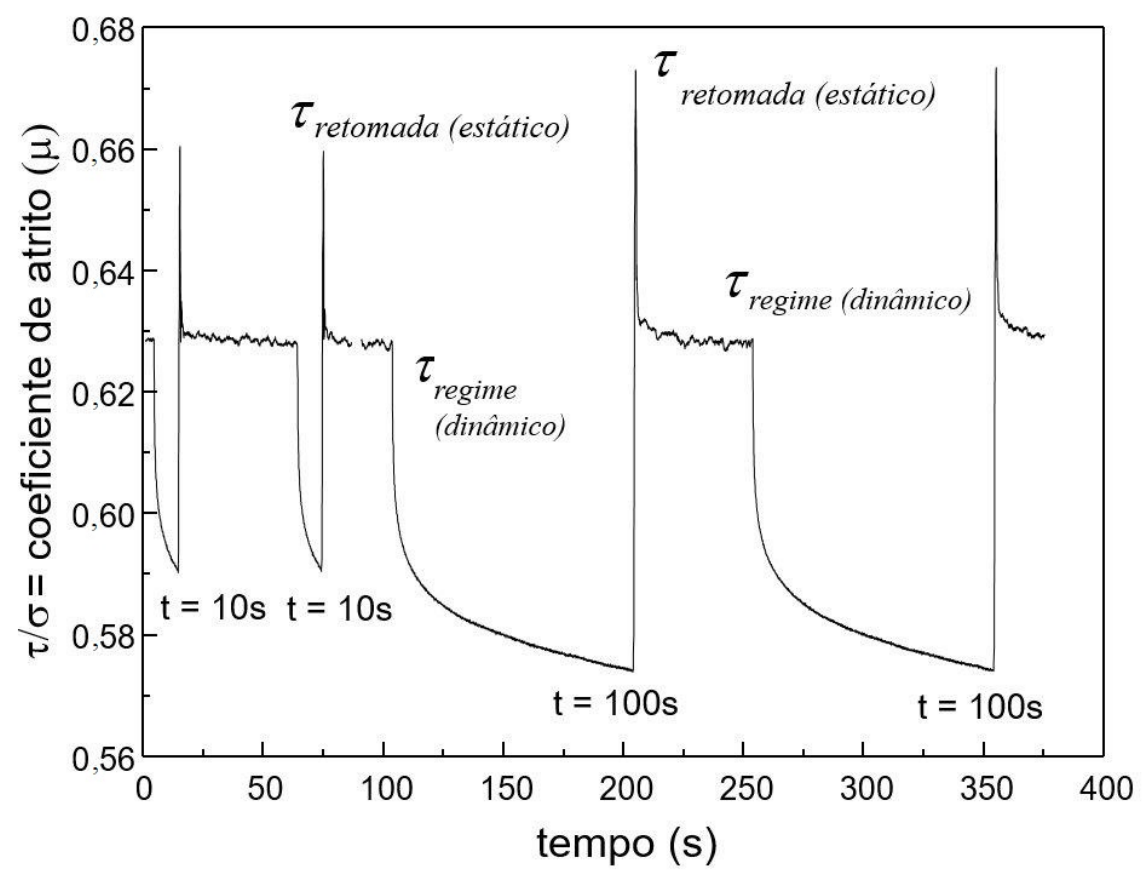

Figura 2. Efeito no incremento da tensão de cisalhamento estática $\left(\tau_{\text {retomada (estático) }}\right)$ para diferentes tempos de repouso $\phi$ (Condotta, 2017). 


\section{Procedimento experimental}

Nos ensaios preliminares da metodologia proposta, foram utilizados quatro tipos de açúcares comerciais: açúcar refinado, açúcar cristal, açúcar confeiteiro (União) e açúcar bruto VHP (very high polarity sugar) padrão exportação (Usina Colombo). A metodologia empregada para a caracterização física desses açúcares secos e a obtenção de suas principais características, como densidades real e aparente (bulk), a distribuição granulométrica e o índice de Hausner, encontra-se disponível na literatura (Santos et al., 2017).

Para tornar o efeito caking mais pronunciável, as amostras de açúcares foram acondicionadas em ambiente de umidade controlada mediante uso de solução saturada de $\mathrm{NaCl}$ a $30{ }^{\circ} \mathrm{C}$ por sete dias, cuja umidade relativa de equilíbrio é de aproximadamente $75 \%$ (Santos et al., 2017).

Os teores exatos de umidade adquiridos pelas amostras de açúcares formam determinados com o auxílio de uma balança com dispositivo de secagem infravermelho (IV-2000, Gehaka).

As principais características dos diferentes tipos de açúcares empregados neste trabalho são apresentadas na Tabela 1.

Tabela 1. Características de cada tipo de açúcar comercial utilizado (Santos et al., 2017).

\begin{tabular}{ccccccc}
\hline $\begin{array}{c}\text { Tipo de } \\
\text { açúcar }\end{array}$ & $\begin{array}{c}\mathbf{D}_{\mathbf{1 0}} \\
(\boldsymbol{\mu \mathbf { m } )}\end{array}$ & $\begin{array}{c}\mathbf{D}_{\mathbf{5 0}} \\
(\boldsymbol{\mu \mathbf { m }})\end{array}$ & $\begin{array}{c}\mathbf{D}_{\mathbf{9 0}} \\
(\boldsymbol{\mu \mathbf { m } )}\end{array}$ & $\begin{array}{c}\text { Umidade } \\
\text { inicial } \mathbf{( \% )}\end{array}$ & $\begin{array}{c}\text { Umidade } \\
\text { induzida }(\mathbf{\%})\end{array}$ & $\begin{array}{c}\boldsymbol{\rho}_{\text {bulk }} \\
\left(\mathbf{g} / \mathbf{c m}^{\mathbf{3}}\right)\end{array}$ \\
\hline VHP & $306 \pm 6,0$ & $696 \pm 7,0$ & $1062 \pm 6,0$ & $0,08 \pm 0,03$ & $0,30 \pm 0,05$ & $0,866 \pm 0,005$ \\
\hline Cristal & $276,0 \pm 0,7$ & $537 \pm 2,0$ & $772 \pm 3,0$ & $0,03 \pm 0,02$ & $0,40 \pm 0,05$ & $0,897 \pm 0,006$ \\
\hline Refinado & $123 \pm 5,0$ & $286 \pm 5,0$ & $704 \pm 16,0$ & $0,31 \pm 0,02$ & $0,90 \pm 0,05$ & $0,777 \pm 0,004$ \\
\hline Confeiteiro & $44,6 \pm 0,4$ & $76,9 \pm 0,7$ & $122 \pm 1,0$ & $0,21 \pm 0,07$ & $1,80 \pm 0,10$ & $0,630 \pm 0,005$ \\
\hline
\end{tabular}

VHP é o açúcar bruto de alta polaridade ("very high polarity sugar"), ainda com a camada de mel que cobre o cristal do açúcar, destinado à exportação. $\mathrm{D}_{10}, \mathrm{D}_{50}$ e $\mathrm{D}_{90}$ são os tamanhos da abertura da peneira que permite a passagem de $10 \%, 50 \%$ e $90 \%$ da massa de amostra, respectivamente.

O teste de cisalhamento instantâneo foi realizado com o auxílio do reômetro de pó FT4 (Freeman Technology), dotado de uma célula de cisalhamento de $50 \mathrm{~mm}$ de diâmetro, aplicando-se tensões normais de consolidação de 3,0, 6,0, 9,0 e 15,0 kPa.

Para cada um dos estados de consolidação, foram mensurados cinco pontos de ruptura nas tensões de aproximadamente $25 \%, 33 \%, 50 \%, 66 \%$ e $80 \%$ da tensão normal de consolidação aplicada, sendo tais testes executados imediatamente após o regime permanente da etapa de consolidação ser atingido.

O ensaio de cisalhamento temporal foi realizado segundo a metodologia de manter a amostra em repouso, tanto sob tensão normal de consolildação quanto a de cisalhamento do estado de regime permanente, no próprio equipamento, conforme a norma D6773-16 (American Society for Testing and Materials, 2016). Foram realizados testes temporais com duas e seis horas de repouso para todas as amostras e, eventualmente, 30 minutos para algumas delas.

Posteriormente, o plano de ruptura temporal foi obtido mediante o deslocamento da curva experimental instantânea até o ponto temporal mensurado, característico do tempo de repouso aplicado. Em alguns casos, foram obtidos pontos temporais em mais de uma tensão normal para o mesmo tempo de repouso, garantindo melhor precisão dos resultados.

A metodologia preditiva proposta neste trabalho consistiu na realização de testes de cisalhamento do tipo stop-and-go apenas do regime permanente na tensão de consolidação das amostras, interrompendo o cisalhamento por diferentes períodos definidos de tempo. Segundo o estudo teórico realizado, a retomada do movimento do sistema implica tensão de cisalhamento momentaneamente superior.

Finalmente, a evolução do valor do pico da tensão de cisalhamento na retomada do movimento foi avaliada em função do tempo de repouso, segundo a expressão de Dietrich-Rice-Ruina. Esse novo valor dependente do tempo foi utilizado como sendo o novo estado de regime permanente de consolidação e, ao realizar a técnica de prorating nos planos de ruptura instantâneo, obteve-se o plano de ruptura temporal preditivo. 


\section{Resultados e discussões}

Inicialmente, foram realizados testes de cisalhamento instantâneo cujos planos de ruptura, bem como os valores numéricos da coesão, obtidos nos ensaios com tensão de consolidação de $9 \mathrm{kPa}$, para cada tipo de açúcar, estão ilustrados na Figura 3:

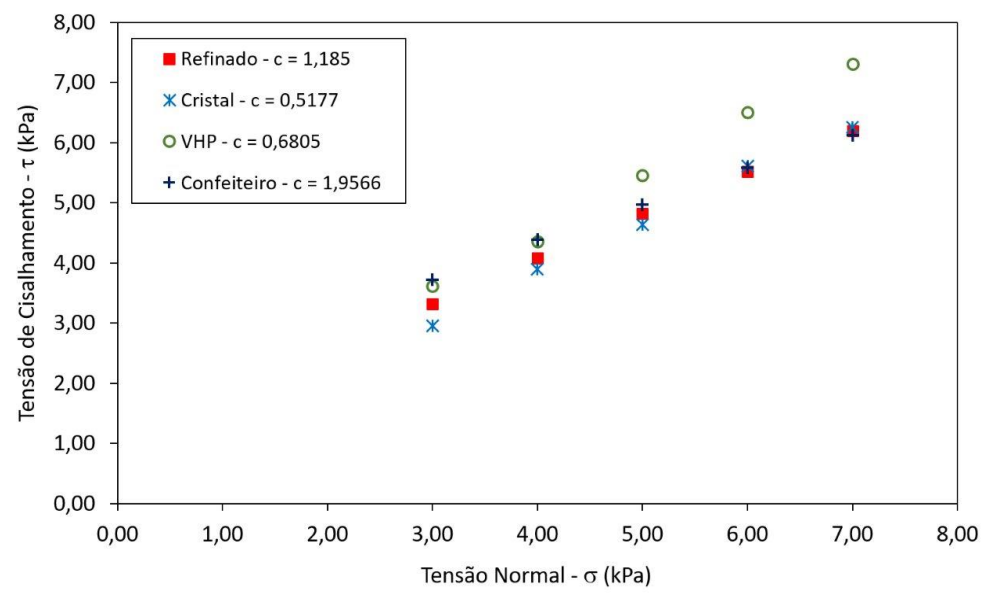

Figura 3. Plano de ruptura e coesão instantâneos dos açúcares a 9 kPa de consolidação.

Sendo a coesão a extrapolação dos planos de ruputra (yield locis), obtidos pela união dos pontos experimentais de ruptura, até a interseção com o eixo da ordenada, verifica-se que o açúcar cristal apresentou o menor valor de coesão dentre todos os açúcares testados, sendo a maior coesão atribuída ao açúcar de confeiteiro.

Ao se comparar os resultados dos testes de cisalhamento com as propriedades dos açúcares, verifica-se que, em linha geral, a coesão aumenta com a redução no tamanho das partículas. Porém, uma coesão levemente superior, mas na mesma ordem de grandeza, é observada para o açúcar VHP em comparação ao açúcar cristal, mesmo o VHP sendo o açúcar de maior granulometria. Ao relacionar a granulometria com a umidade adsorvida, constata-se que as amostras com menor tamanho de partículas são as que apresentaram os maiores teores de umidade. Conclui-se, então, que a umidade é promotora da coesão desenvolvida nas amostras de açúcar, conforme constatado nos trabalhos de Chávez Montes et al. (2011), Juliano \& Barbosa-Cánovas (2010) e Fürll \& Hoffmann (2013).

Em seguida, procedeu-se à obtenção dos planos de ruptura temporais experimentais a duas e seis horas de repouso. Conforme descrito na literatura, a obtenção de apenas um único ponto experimental é suficiente para estimar o plano completo, adotando-se a mesma inclinação do plano de ruptura intantâneo, pois infere-se que o material apresenta o mesmo coeficiente de atrito.

Ao adotar esse conceito, ensaios do tipo stop-and-go foram realizados para os quatro tipos de açúcares apenas na tensão de consolidação de $9 \mathrm{kPa}$. Sendo o coeficiente de atrito a tensão de cisalhamento medida pela tensão normal aplicada, a tensão de cisalhamento no regime estacionário dos demais estágios de consolidação foi estimada empregando-se o mesmo coeficiente de atrito obtido nos ensaios de $9 \mathrm{kPa}$. Uma vez que o coeficiente de atrito pode ser corrigido para cada tempo de repouso segundo a expressão de Dietrich-Rice-Ruina, os coeficientes das demais tensões também foram estimados.

Diante do fato de que o equipamento permanece inutilizável durante o repouso da amostra e que é necessário realizar testes para cada uma das cinco tensões de ruptura, para todas as quatro tensões de consolidação aplicadas, para cada amostra de açúcar, para cada tempo de repouso, reduziram-se a determinação do plano de ruptura experimental para apenas duas tensões normais e os períodos de repouso 
para duas e seis horas apenas. Além disso, conforme já explanado, os testes stop-and-go foram realizados apenas para a tensão de $9 \mathrm{kPa}$.

As Figuras de 4 a 7 ilustram os planos de ruptura temporais experimentais e os preditos pela metodologia proposta, sob tensão normal de $9 \mathrm{kPa}$.

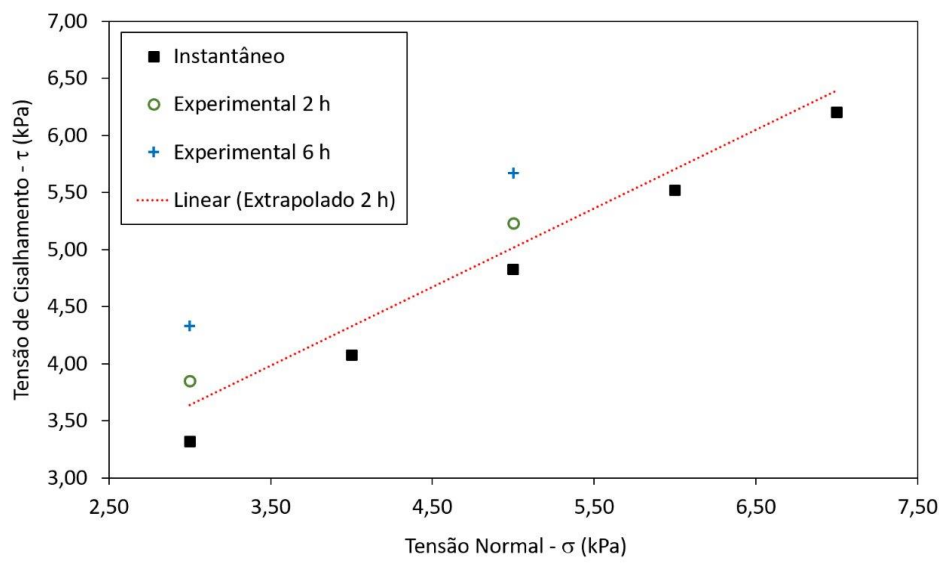

Figura 4. Planos de ruptura instantâneo e temporais para o açúcar refinado.

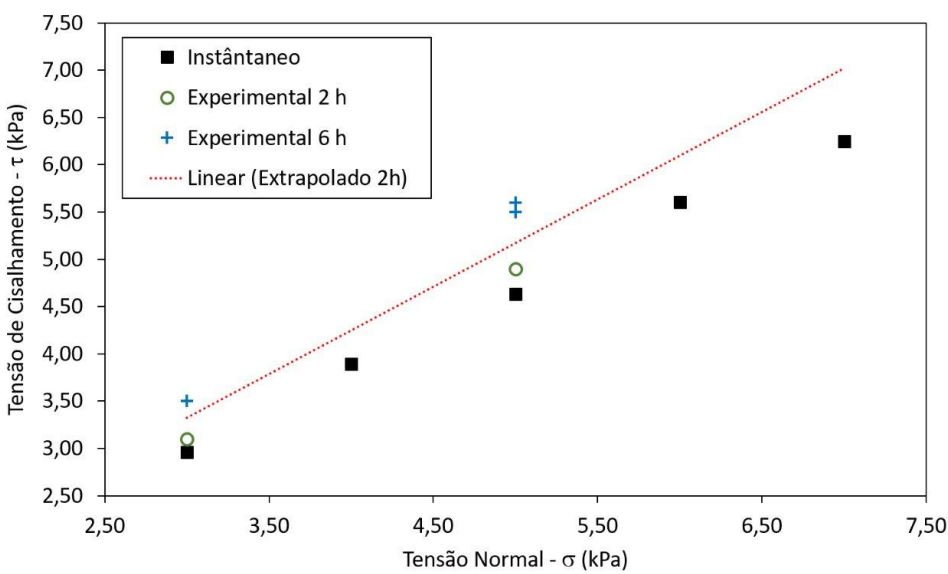

Figura 5. Planos de ruptura instantâneo e temporais para o açúcar cristal.

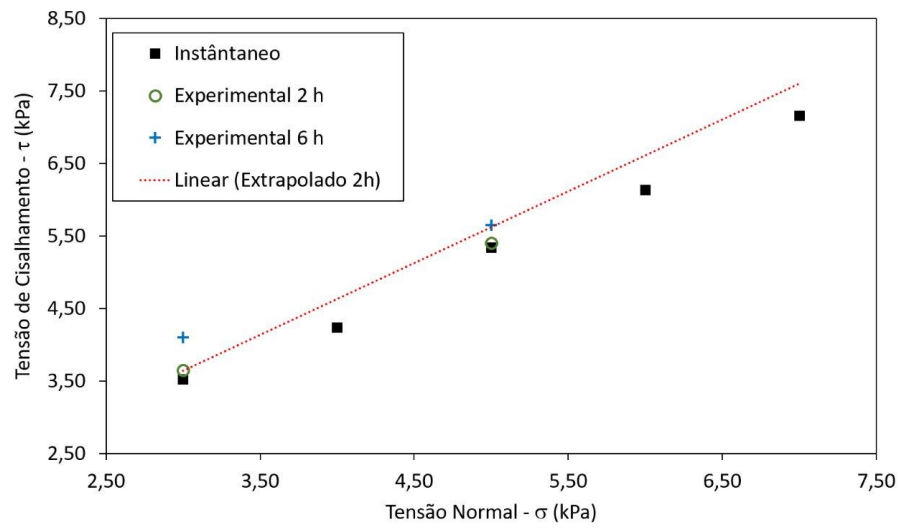

Figura 6. Planos de ruptura instantâneo e temporais para o açúcar VHP. 


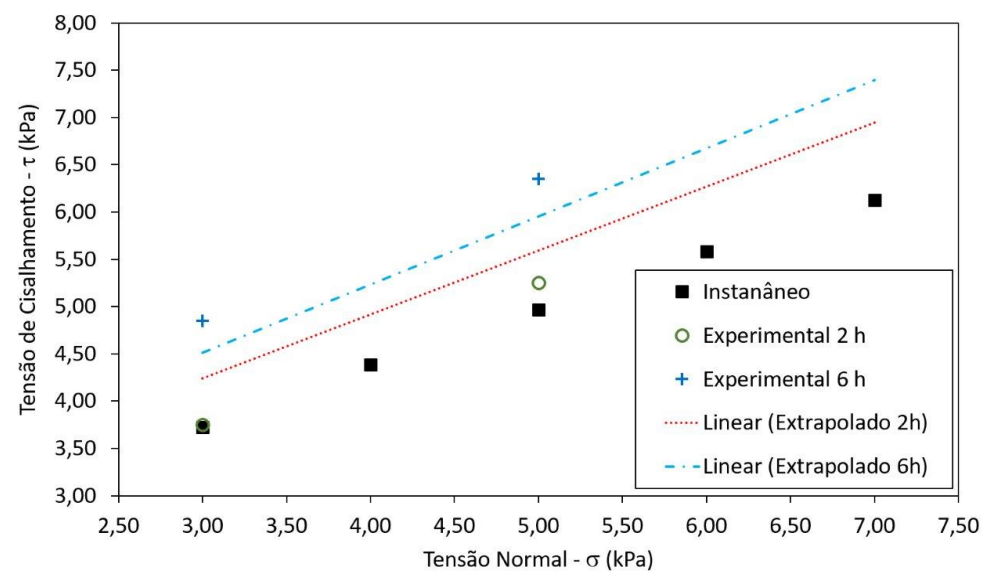

Figura 7. Planos de ruptura instantâneo e temporais para o açúcar de confeiteiro.

Verifica-se que os pontos de ruptura temporais experimentais são distintos dos pontos instantâneos, para todos os tipos de açúcar, demonstrando uma clara evolução dos planos de ruputura com o aumento do tempo de repouso, porém de forma menos expressiva para o açúcar VHP (Figura 6). Observa-se ainda que os planos de ruptura temporais para os açúcares refinado e cristal são exatamente paralelos aos seus respectivos planos instantâneos (Figuras 4 e 5), confirmando a hipótese de que o coeficiente de atrito das amostras secas e úmidas, indicada pela inclinação dos planos, permanece inalterada. Já para o açúcar VHP, verifica-se uma convergência dos planos na região de baixas tensões normais, e na região de elevadas tensões para o açúcar de confeiteiro, em razão das possíveis modificações das condições desses tipos de açúcar durante o período em que permaneceram em repouso (umidade, temperatura etc.).

Segundo a metodologia proposta, constata-se ainda que os valores extrapolados se mostraram superiores aos planos instantâneos e próximos dos planos de ruptura temporais experimentais. Entretanto, para o açúcar refinado, o plano predito para duas horas de repouso é subestimado em relação ao experimental (Figura 4), enquanto para os demais tipos de açúcar é ligeiramente superestimado, porém inferior ao experimental de seis horas em todos os casos, mostrando que a metolodoliga é sensível às variações das propriedades das amostras ao longo do tempo de repouso.

Mais resultados consistentes entre os planos de ruptura temporais preditos e experimentais, sob as demais tensões de consolidação, também foram obtidos e estão ilustrados nas Figuras 8 a 11:

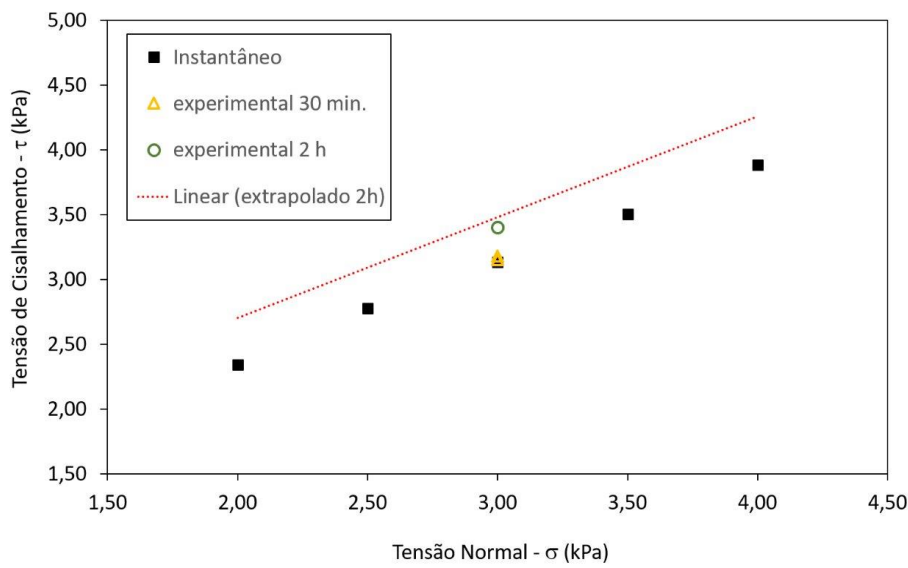

Figura 8. Planos de ruptura instantâneo e temporal para o açúcar refinado a $6 \mathrm{kPa}$. 


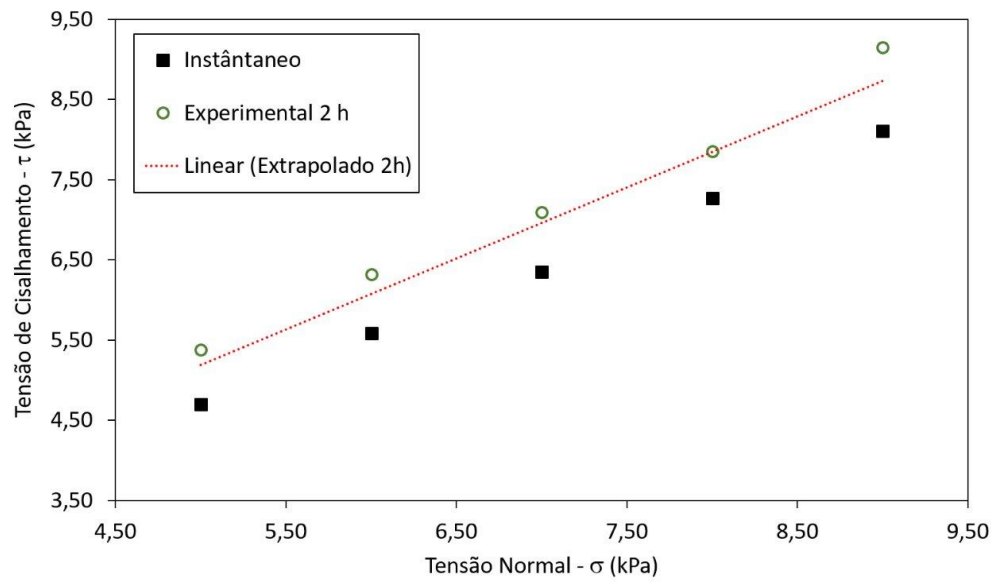

Figura 9. Planos de ruptura instantâneo e temporal para o açúcar cristal a $15 \mathrm{kPa}$.

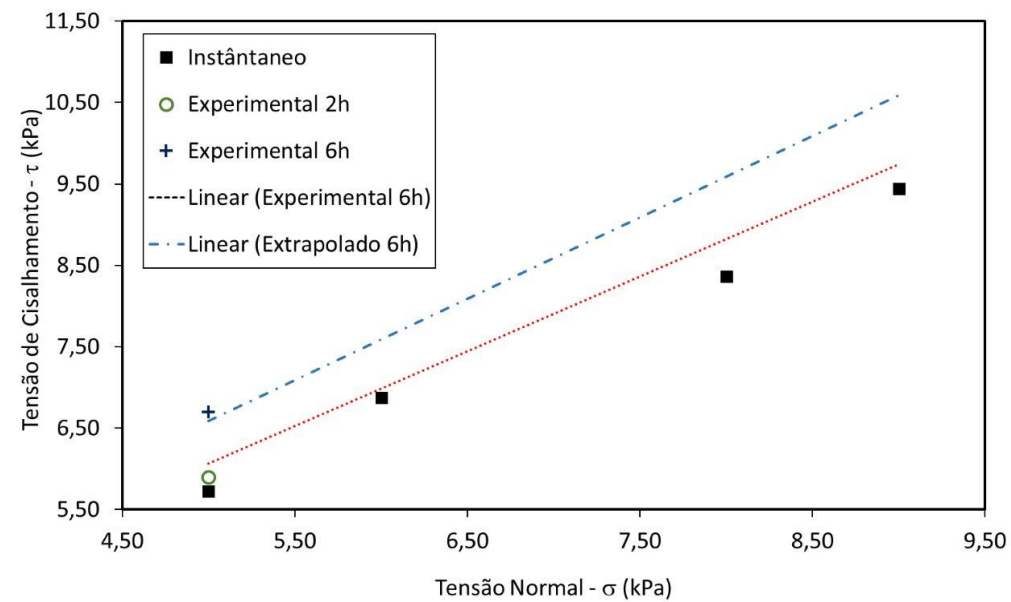

Figura 10. Planos de ruptura instantâneo e temporal para o açúcar VHP a 15 kPa.

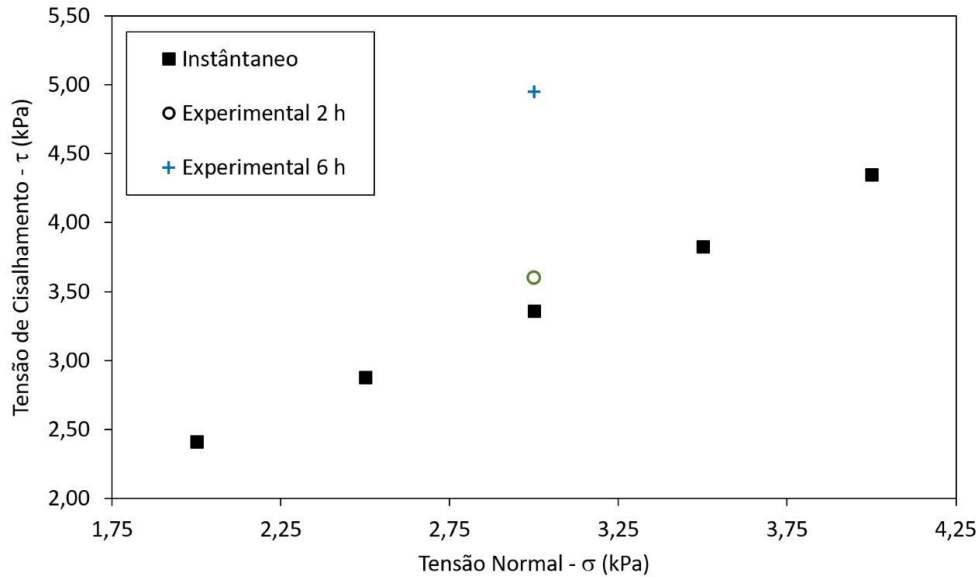

Figura 11. Planos de ruptura instantâneo e pontos de ruptura temporal para o açúcar de confeiteiro a $6 \mathrm{kPa}$.

Mais uma vez, observam-se evolução e distinção consideráveis entre os planos de ruptura instantâneos e os preditos para duas e seis horas de repouso, como também proximidade entre os plano preditos pela técnica proposta a partir de seus respectivos valores experimentais. Tal observação se torna mais evidente no ensaio 
temporal completo realizado para o açúcar Cristal, o qual contemplou a realização dos cinco pontos experimentais de ruptura para o período de duas horas de repouso (Figura 9).

A fim de verificar a sensibilidade da metodologia empregada, estimou-se um ponto de ruptura experimental para o período de 30 minutos para o açúcar refinado (Figura 8). Obteve-se uma pequena elevação da tensão de cisalhamento, mas inferior ao período de duas horas, demonstrando que a metodologia é satisfatória.

Finalmente, observou-se que, dentre todos os tipos de açúcares, o de confeiteiro foi o que apresentou mais tendência ao efeito caking, dada sua maior coesão (Figura 3) e maior discrepância entre os planos de ruptura nos tempos de duas e seis horas (Figuras 7 e 11). Já o açúcar VHP, apesar de apresentar a segunda maior coesão nos ensaios instantâneos, foi o que apresentou menos sensibilidade ao efeito caking, seguido pelo açúcar cristal, em razão das menores evoluções dos planos de ruputa temporais observados a duas e seis horas para esses dois tipos de açúcares. Tais constatações são coerentes com a experiência pessoal que todos nós possuímos ao manusear esses diferentes tipos de açúcares nos afazeres domésticos.

\section{Conclusões}

A determinação de planos de ruptura de amostras mantida em repouso durante longos períodos ainda é repleta de desafios. Alterações nas condições da amostra ou mesmo do ambiente durante a realização do ensaio podem influenciar nos resultados. Desta forma, a metodologia preditiva proposta para estimar o efeito caking de longos períodos, partindo de extrapolações de dados experimentais obtidos em curtos períodos de repouso, em que essas alterações são mínimas, mostrou ser uma alternativa interessante.

Os resultados para os quatro tipos de açúcares úmidos apresentaram uma boa aproximação entre os planos temporais experimentais e os preditos, além de clara distinção entre os diferentes períodos de repouso avaliados.

Apesar dos primeiros resultados encorajadores, mais testes com diferentes materiais particulados são necessários para validar a metodologia preditiva proposta, mas que, se comprovada, será uma boa ferramenta para as indústrias.

\section{Nomenclaturas}

\begin{tabular}{|c|c|c|}
\hline$a$ e $b$ & Coeficientes da expressão de Dieterich-Rice-Ruina & - \\
\hline$c$ & Coesão & $\mathrm{kPa}$ \\
\hline$D$ & Distância de deslocamento durante o cisalhamento & $\mathrm{mm}$ \\
\hline$f_{c}$ & Tensão normal de não confinamento & $\mathrm{kPa}$ \\
\hline$F F$ & Função de fluxo & - \\
\hline$\varphi_{i}$ & Ângulo de atrito interno & $\circ$ \\
\hline$\varphi_{e f}$ & Ângulo efetivo de atrito interno & $\circ$ \\
\hline$V_{0}$ e $V$ & Velocidades de cisalhamento inferior e superior, respectivamente & $\mathrm{mm} / \mathrm{s}$ \\
\hline$\phi$ & Tempo de cisalhamento & $\mathrm{S}$ \\
\hline$\mu_{0}$ & Coeficiente de atrito estático genérico sob velocidade $V_{0}$ & - \\
\hline$\mu_{\max }$ & Coeficiente de atrito estático máximo (na retomada do movimento) & - \\
\hline$\mu_{s s}$ & Coeficiente de atrito dinâmico (regime estacionário) & - \\
\hline$\rho$ bulk & Densidade aparente (de bulk) da amostras & $\mathrm{g} / \mathrm{cm}^{3}$ \\
\hline$\sigma$ & Tensão normal & $\mathrm{kPa}$ \\
\hline$\sigma_{1}$ & Tensão normal principal (máxima) & $\mathrm{kPa}$ \\
\hline$\sigma_{c}$ & Tensão normal de consolidação & $\mathrm{kPa}$ \\
\hline$\sigma_{N 1,} \sigma_{N 2,} \sigma_{N 3}$ & Tensão normal aplicada nos ensaios $1,2,3$, respectivamente & $\mathrm{kPa}$ \\
\hline$\tau$ & Tensão de cisalhamento & $\mathrm{kPa}$ \\
\hline$\tau_{\exp i}$ & Tensão de cisalhamento do experimento i obtida experimetalmente & $\mathrm{kPa}$ \\
\hline$\tau^{\prime} \exp i$ & Tensão de cisalhamento do experimento i corrigida & $\mathrm{kPa}$ \\
\hline$\tau_{c}$ & Tensão de cisalhamento sob a tensão normal de consolidação & $\mathrm{kPa}$ \\
\hline$\tau_{c \exp i}$ & Tensão de cisalhamento sob a tensão de consolidação do experimento i & $\mathrm{kPa}$ \\
\hline$\tau_{c \text { médio }}$ & Tensão de cisalhamento média de todo os ensaios sob $\sigma_{c}$ & $\mathrm{kPa}$ \\
\hline
\end{tabular}




\section{Referências}

American Society for Testing and Materials - ASTM International. (2014). Standard test method for shear testing of bulk solids using the Jenike shear cell (D6128-14). Pennsylvania: ASTM International.

American Society for Testing and Materials - ASTM International. (2016). Standard test method for shear testing of bulk solids using Schulze ring shear tester (D6773-16). Pennsylvania: ASTM International.

Brasil. Ministério da Agricultura, Pecuária e Abastecimento. (2017). Cana-de-açúcar. Brasília. Recuperado em 18 de março de 2019, de http://www.agricultura.gov.br/vegetal/culturas/cana-de-acucar

Calvert, G., Curcic, N., Redhead, C., Ahmadian, H., Owen, C., Beckett, D., \& Ghadiri, M. (2013). A new environmental bulk powder caking tester. Powder Technology, 249, 323-329. http://dx.doi.org/10.1016/j.powtec.2013.08.037

Campos, M. M., \& Ferreira, M. C. (2013). A comparative analysis of the flow properties between two alumina-based dry powders. Advances in Materials Science and Engineering, 2013, 519846. http://dx.doi.org/10.1155/2013/519846

Chávez Montes, E., Ardila Santamaría, N., Gumy, J.-C., \& Marchal, P. (2011). Moisture-induced caking of beverage powders. Journal of the Science of Food and Agriculture, 91(14), 2582-2586. PMid:21674508. http://dx.doi.org/10.1002/jsfa.4496

Condotta, R. (2017). Coulabilité des poudres cohesives. Paris: Presses Académiques Francophones. 272p.

Condotta, R., \& Machado, C. S. (2015). Effect of particles size on flowability properties of particulate solids. Récents Progrès en Génie des Procédés, (107), 1-9.

De Ryck, A., Condotta, R., \& Lubert, M. (2003). Interrupted shear of granular media. The European Physical Journal E, 11(2), 159-167. PMid:15011056. http://dx.doi.org/10.1140/epje/i2002-10153-6

Dieterich, J. H. (1979). Modeling of rock friction: 1. Experimental results and constitutive equations. Journal of Geophysical Research. Solid Earth, 84(B5), 2161-2168. http://dx.doi.org/10.1029/JB084iB05p02161

Dieterich, J. H., \& Conrad, G. (1984). Effect of humidity on time and velocity dependent friction in rocks. Journal of Geophysical Research. Solid Earth, 89(B6), 4196-4202. http://dx.doi.org/10.1029/JB089iB06p04196

Fitzpatrick, J. J., \& Ahrné, L. (2005). Food powder handling processing: Industry problems, knowledge barriers and research opportunities. Chemical Engineering and Processing: Process Intensification, 44(2), 209-214. http://dx.doi.org/10.1016/j.cep.2004.03.014

Fürll, C., \& Hoffmann, T. (2013). The influence of the granulometric condition on the flow characteristics of shredded grain products in their dependence on the duration of storage. Powder Technology, 235, 307-311. http://dx.doi.org/10.1016/j.powtec.2012.10.040

Hartmann, M., \& Palzer, S. (2011). Caking of amorphous powders: Material aspects, modelling and applications. Powder Technology, 206(1-2), 112-121. http://dx.doi.org/10.1016/j.powtec.2010.04.014

Jenike, A. W. (1964). Storage and flow of solids (Bulletin, No. 123). Utah: University of Utah.

Johanson, K. (2009). Effect of particle shape on unconfined yield strength. Powder Technology, 194(3), 246-251. http://dx.doi.org/10.1016/j.powtec.2009.05.004

Juliano, P., \& Barbosa-Cánovas, G. V. (2010). Food powders flowability characterization: Theory, methods, and applications. Annual Review of Food Science and Technology, 1(1), 211-239. PMid:22129336. http://dx.doi.org/10.1146/annurev.food.102308.124155

Lopes-Neto, J. P., Nascimento, J. W. B., Silva, V. R., \& Lopes, F. F. M. (2007). Flow properties and flowability characteristics of poultry rations for design of silos. Ciência e Agrotecnologia, 31(3), 851-859.

Lubert, M., De Ryck, A., \& Dodds, J. A. (2001). Evaluation of the mechanical properties of powder for storage. In A. Levy \& H. Kalman (Eds.), Handbook of conveying and handling of particulate solids (pp. 65-72). Elsevier Science. http://dx.doi.org/10.1016/S0167-3785(01)80009-9.

Mehos, G. (2016). Prevent caking on bulk solids. Chemical Engineering and Processing, 4, 48-55.

Rice, J. R., \& Ruina, A. L. (1983). Stability of steady frictional slipping. Journal of Applied Mechanics, 50(2), 343-349. http://dx.doi.org/10.1115/1.3167042

Rogé, B., \& Mathlouthi, M. (2003). Caking of white crystalline sugar. International Sugar Journal, 105(1251), 128-136.

Santos, L. C., Condotta, R., \& Ferreira, M. C. (2017). Flow properties of coarse and fine sugar powders. Journal of Food Process Engineering, 41(2), 1-10.

Schwedes, J. (2003). Review on testers for measuring flow properties of bulk solids. Granular Matter, 5(1), 1-43. http://dx.doi.org/10.1007/s10035-002-0124-4

Silva, P. M., \& Condotta, R. (2017). Avaliação do efeito da umidade nas propriedades de escoamento de diferentes tipos de açúcar. In Blucher Chemical Engineering Proceedings: Anais do XII Congresso Brasileiro de Engenharia Química em Iniciação Científica. São Carlos: UFSCar. http://dx.doi.org/10.5151/chemeng-cobeqic2017-435

Zafar, U., Vivacqua, V., Calvert, G., Ghadiri, M., \& Cleaver, J. A. S. (2017). A review of bulk powder caking. Powder Technology, 313, 389-401. http://dx.doi.org/10.1016/j.powtec.2017.02.024

Financiamento: Centro Universitário da Fundação Educacional Inaciana Pe. Sabóia de Medeiros. 